\title{
Variantes anatómicas relevantes en tomografía computarizada de cavidades perinasales
}

\author{
Remarkable anatomic variations on paranasal CT scans \\ Rodrigo Cabezón $\mathbf{A}^{\mathbf{1}}$, Ricardo Valdés $\mathbf{S}^{\mathbf{1}}$, Hayo Breinbauer $\mathrm{K}^{\mathbf{1}}$, Constanza Ramírez $\mathrm{R}^{\mathbf{2}}$, \\ Carolina Grau $\mathrm{L}^{3}$, Rodrigo Iñíguez $\mathrm{C}^{1}$.
}

\begin{abstract}
RESUMEN
Introducción: La anatomía ósea de las cavidades perinasales (CPN) es altamente variable, pudiendo ser causa o factor predisponente de patología rinosinusal, o servir de reparo anatómico para la cirugía endoscópica nasal (CEN).

objetivos: Conocer la frecuencia de variaciones anatómicas en pacientes que consultan en nuestra Red de Salud.

Material y método: Revisión retrospectiva de todas las tomografías computarizadas (TC) de CPN, realizadas en el Servicio de Radiología de la Red de Salud UC entre abril y junio de 2009.

Resultados: Se encontraron las distintas variantes anatómicas en frecuencias similares a las descritas en la literatura internacional. Se estudió también la trayectoria de la arteria etmoidal anterior (AEA), y la configuración del techo etmoidal, utilizando la clasificación de Keros.

Conclusiones: La frecuencia de variaciones anatómicas encontrada en nuestra revisión es similar a la descrita en la literatura internacional. La mayoría de nuestros pacientes presenta configuración de techo etmoidal tipo Keros I, hallazgo diferente al reportado en otras series. El estudio metódico de las variantes anatómicas en TC de CPN nos permitiría evitar complicaciones quirúrgicas.

Palabras clave: Variantes anatómicas etmoidales, TC de CPN, cirugía endoscópica nasal, Keros.
\end{abstract}

\section{ABSTRACT}

Introduction: Paranasal sinus anatomy is quite variable. Some variations are involved in rinosinusal pathology, others are landmarks of FESS (functional endoscopic sinus surgery).

Aim: To determine the frecuency of paranasal anatomic variations in our consulting population.

Material and method: Retrospective revision of all paranasal CT scans achieved in our Radiology Service during a two month period during 2009.

\footnotetext{
Médico. Departamento de Otorrinolaringología, Hospital Clínico de la Pontificia Universidad Católica de Chile.

2 Interna de Medicina. Hospital Clínico de la Universidad de Chile.

3 Interna de Medicina. Hospital Clínico de la Pontificia Universidad Católica de Chile.
} 
Results: We found most of ethmoid anatomic variations, with similar frecuencies as described in literature. We also studied anterior ethmoidal artery anatomy, and ethmoid roof configuration, using Keros classification

Conclusions: We found, in general, similar frecuencies as described in foreign series. More than half of our patients had Keros I ethmoid roof configuration type, a differing result from most of other series. Every ENT surgeon should be trained in routine search of these variations, helping to avoid possible surgical complications.

Key words: Ethmoid variations, paranasal CT scans, sinus endoscopic surgery, Keros.

\section{INTRODUCCIÓN}

La anatomía ósea de las cavidades paranasales (CPN) presentan múltiples variantes, que pueden ser causa 0 factor predisponente de patología rinosinusal. Éstas pueden ser reparos de gran importancia en la cirugía endoscópica nasal (CEN). Es por esto que su detección es útil para programar cirugías y de esta manera prevenir complicaciones.

En la literatura se encuentran múltiples estudios descriptivos de la frecuencia de variantes anatómi- cas tanto en pacientes sanos como con patología rinosinusal. Estos estudios muestran resultados muy disímiles entre sí (Tabla 1). Sin embargo, no existen trabajos descriptivos de la frecuencia de estas variantes en población nacional.

Existe controversia si es que la existencia de variantes anatómicas óseas aumentaría el riesgo de presentar enfermedad sinusal. Bolger ${ }^{1}$ comparó pacientes con patología versus controles sanos, sin encontrar diferencias significativas, lo que apoyaría la hipótesis de una patogenia multifactorial en el desarrollo de la patología rinosinusal2.

Tabla 1. Frecuencia de variaciones anatómicas descritas en la literatura

\begin{tabular}{|c|c|c|}
\hline Variante anatómica & Frecuencia & Autor \\
\hline \multirow[t]{2}{*}{ Septodesviación } & $45 \%-85 \%$ & Kennedy, Zinreich ${ }^{1}$ \\
\hline & $36 \%$ & Arslan et $a^{5}$ \\
\hline Cornete medio paradojal & $3 \%$ & Arslan et al ${ }^{5}$ \\
\hline \multirow[t]{4}{*}{ Concha bulosa } & $33 \%$ & Aguilera et al ${ }^{12}$ \\
\hline & $14 \%$ & González Rosado ${ }^{33}$ \\
\hline & $35 \%$ & Nouraei et a $\left.\right|^{11}$ \\
\hline & $30 \%$ & Arslan et al ${ }^{5}$ \\
\hline \multirow[t]{3}{*}{ Cornete medio secundario } & $2,50 \%$ & Kantarci ${ }^{2}$ \\
\hline & $0,80 \%$ & Aksungur et a ${ }^{34}$ \\
\hline & $1,07 \%$ & González Rosado ${ }^{33}$ \\
\hline \multirow[t]{2}{*}{ Unciforme neumatizada } & $0,40 \%$ & Kennedy, Zinreich ${ }^{1}$ \\
\hline & $2,50 \%$ & Bolger et al ${ }^{31}$ \\
\hline \multirow[t]{3}{*}{ Celdilla de Onodi } & $10,40 \%$ & Basic et a $\left.\right|^{32}$ \\
\hline & $8 \%-14 \%$ & Kennedy, Zinreich ${ }^{1}$ \\
\hline & $12 \%$ & Arslan et $a^{5}$ \\
\hline \multirow[t]{3}{*}{ Celdilla de Haller } & $10 \%$ & Kennedy, Zinreich ${ }^{1}$ \\
\hline & $18 \%$ & Kantarci ${ }^{2}$ \\
\hline & $21,20 \%$ & Basic et al ${ }^{32}$ \\
\hline \multirow[t]{3}{*}{ Agger Nassi } & $65 \%$ & Davis $^{2}$ \\
\hline & $89 \%$ & Van Alyea ${ }^{2}$ \\
\hline & $47 \%$ & Kantarci² \\
\hline \multirow{4}{*}{$\begin{array}{l}\text { Techo etmoidal } \\
\text { (Keros I, II, III) }\end{array}$} & $12 \%, 70 \%, 18 \%$ & Keros $^{27}$ \\
\hline & $20 \%, 16 \%, 64 \%$ & Arslan et al $\left.\right|^{5}$ \\
\hline & $29 \%, 60 \%, 11 \%$ & González Rosado ${ }^{33}$ \\
\hline & $81,5 \%, 18 \%, 0,5 \%$ & Josefino et al ${ }^{29}$ \\
\hline
\end{tabular}


El objetivo de este trabajo fue conocer la frecuencia de distintas variantes anatómicas óseas de nariz y CPN en pacientes que consultan en nuestra Red de Salud, y comparar estos resultados con los descritos en la literatura médica internacional.

\section{MATERIAL Y MÉTODO}

Se realizó una revisión retrospectiva de todas las tomografías computarizadas (TC) de CPN realizada en el Servicio de Radiología de la Red de Salud UC (excluido San Carlos de Apoquindo), desde el 20 de abril hasta el 20 de junio de 2009. Se identificaron 165 TC, excluyendo del análisis los TC repetidos, posquirúrgicos de CEN y tumores de CPN.

Se revisaron finalmente $142 \mathrm{TC}$, con todos los cortes axiales y coronales disponibles, con un grosor de corte de 2 a $3 \mathrm{~mm}$, por 4 revisores independientes, siendo éstos médicos residentes 0 miembros ORL de nuestro departamento. Para determinar la presencia de cada variante anatómica, se utilizó el criterio consensuado de los 4 revisores (Figura 1).

\section{RESULTADOS}

Con respecto a la distribución por género, 51\% de las TC correspondió a pacientes de sexo masculino y $49 \%$ a sexo femenino. El $90 \%$ de las TC analizadas correspondieron a adultos y $10 \%$ a población pediátrica (menores de 15 años).
De las TC analizadas en la revisión, el 46\% fueron solicitados por ORL, seguido por $9 \%$ de TC solicitadas por medicina familiar o medicina interna. El $26 \%$ de las TC no tenía ningún médico solicitante consignado (Figura 2).

Los resultados están expuestos en la Tabla 2.

\section{DISCUSIÓN}

En porcentajes absolutos, la variación anatómica más frecuente fue la presencia de celdillas de Agger Nassi (82\%), definida como la presencia de celdillas etmoidales anteriores a la inserción anterior de los cornetes medios al revisar los cortes coronales de anterior a posterior. La prevalencia de este hallazgo que ha sido reportada, varía de manera significativa dependiendo de los autores, desde 10\%-15\% descrito por Messerklinger ${ }^{1}$ en 1967, hasta 89\% descrito por Van Alyea². Por su alta frecuencia, debería llevarnos a considerarla en la práctica como una "constante anatómica», siendo su ausencia una variación. La presencia de las celdillas de Agger Nassi de gran tamaño podría relacionarse con la sinusitis crónica frontal, ya que puede llegar a impedir el drenaje del seno frontal al formar parte de la pared anterior del receso frontal|3,4. Los cortes sagitales de la TC de CPN permiten obtener un conocimiento detallado de la anatomía del conducto nasofrontal, lo que es tremendamente útil para el abordaje endoscópico del seno frontal.

La desviación septal fue igualmente encontrada en la mayoría de las TC analizadas (72\%), siendo

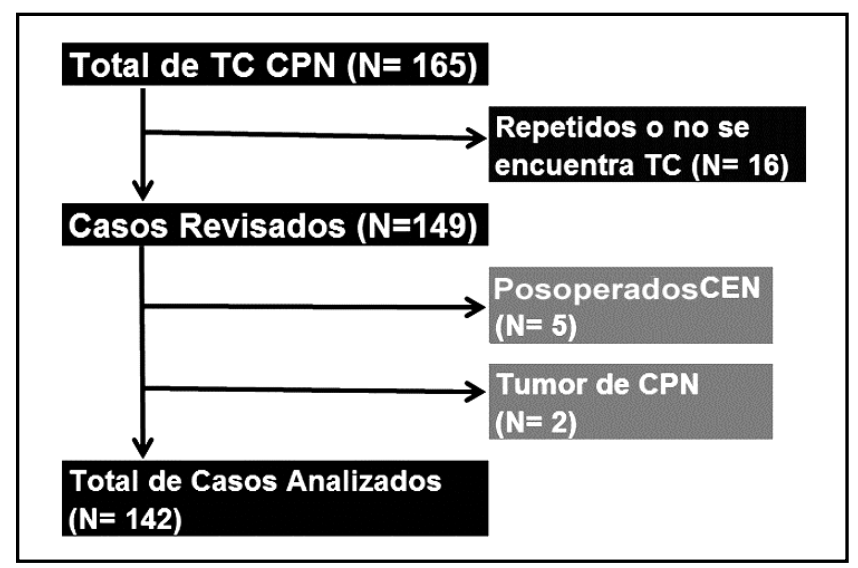

Figura 1. Material y método. 


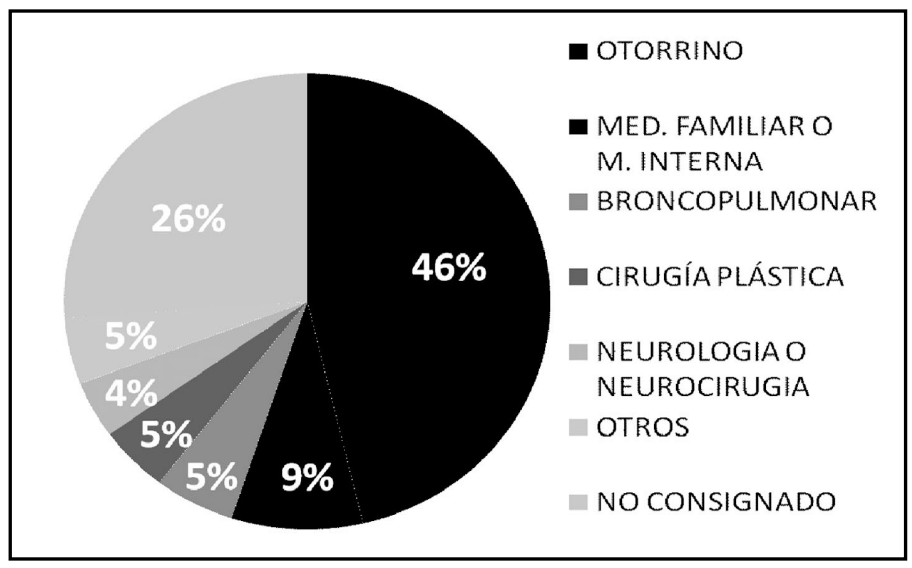

Figura 2. Distribución de las TC de CPN según especialidad solicitante del examen.

Tabla 2. Tabla resumen de nuestros resultados

\begin{tabular}{|lr|}
\hline Variaciones anatómicas TC de CPN & $\%$ \\
\hline 1) Tabique & \\
Septodesviación & 72,53 \\
Espolón & 34,51 \\
2) Cornete medio & \\
$\quad$ Concha bulosa & 30,28 \\
Paradojal & 9,86 \\
Supernumerario & 1,41 \\
3) Otros cornetes & \\
Neumatización cornete superior & 4,93 \\
4) Proceso unciforme & \\
$\quad$ Neumatización & 0 \\
5) Variantes etmoidales & \\
$\quad$ Agger Nassi & \\
Haller & 82,39 \\
Onodi & 10,56 \\
6) Hipoplasia/aplasia senos* & 13,38 \\
Maxilar & \\
Frontal & 3,52 \\
7) Techo etmoidal (Keros) & 12,68 \\
Keros I & \\
Keros II & \\
Keros III & \\
8) Trayectoria arteria etmoidal anterior** & \\
«Alta» & \\
«Baja» & 62,27 \\
\hline
\end{tabular}

${ }^{*}$ Se excluyeron los TC de CPN pediátricos. * *Porcentajes corresponden al total de casos analizables $(N=59)$.

ésta definida operacionalmente por nuestro equipo como una desviación del tabique que pareciese significativa al observador. Las frecuencias infor- madas en la literatura internacional son variables. El estudio de $\operatorname{Arslan}^{5}$, evidencia $36 \%$, el de J. Jiménez-Castellanos $58 \%{ }^{6}$, hasta uno de los trabajos clásicos de Kennedy, donde describe hasta $85 \%{ }^{7}$. Clásicamente a esta variación se le ha asignado responsabilidad en la ocurrencia de la rinosinusitis crónica. Sin embargo, la evidencia es

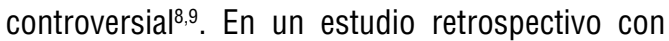
1.452 pacientes, no habría diferencia significativa en la incidencia de rinosinusitis crónica, sólo se podría identificar como un factor patogénico a las desviaciones severas ${ }^{10}$.

Con respecto a variaciones anatómicas del cornete medio, encontramos $30 \%$ de conchas bulosas (definida como la neumatización parcial 0 completa del cornete medio), y $10 \%$ de cornetes paradojales, resultados concordantes con otros estudios $^{5,11,12}$. Ambas variaciones pueden contribuir en la obstrucción del complejo osteomeatal (COM), y por tanto, en la patogenia de la rinosinusitis aguda recurrente y la rinosinusistis crónica ${ }^{2}$. Asimismo, encontramos 2 casos $(1,4 \%)$ de cornetes medios supernumerarios (Figura 3), entidad descrita por primera vez por Khanobthamchai en $1991^{13,34}$, y que puede ser confundido con un pólipo 0 un osteoma que obstruye el COM si no se cuenta con una TC con cortes coronales. Dentro de su diagnóstico diferencial, debemos considerar también la posible horizontalización de la apófisis unciforme.

La neumatización del proceso unciforme es una entidad poco frecuente. Ésta ha sido descrita 
por varios autores (Kennedy, Zinreich $(0,4 \%)$, Bolger $(2,5 \%))$, como una variación que produce obstrucción del infundíbulo. La neumatización del proceso unciforme, junto a otras alteraciones de la ventilación del COM, como una celdilla infraorbitaria, podrían ser factores causales de sinusitis maxilares unilaterales recurrentes ${ }^{2}$. Nosotros no encontramos ningún caso en nuestra serie, lo que es compatible con las bajas frecuencias descritas por los autores previamente citados.

Observamos 4,9\% de cornetes superiores neumatizados, variación anatómica, que de no contar con una TC de CPN es muy difícil de evaluar, ya que es difícil de observar endoscópicamente. Algunos autores lo asocian a la cefalea por puntos de contacto 0 rinógena, diagnóstico bastante controversial, caracterizado por la presencia de cefalea que revierte con el uso de vasoconstrictores y anestésicos tópicos nasales ${ }^{14}$. La existencia de un cornete superior de gran tamaño generaría un punto de contacto mucoso en la zona más estrecha de la fosa nasal, provocando este cuadro ${ }^{15}$. Por otra parte, existen diversos autores que cuestionan la existencia de esta patología, y que incluso asocian la presencia de cefalea con una menor probabilidad de presentar una rinosinusitis crónica ${ }^{16-19}$.

La hipoplasia unilateral de senos paranasales, se definió arbitrariamente como la diferencia en $50 \%$ o más de volumen con respecto al seno contralateral, ya que la asimetría de senos es

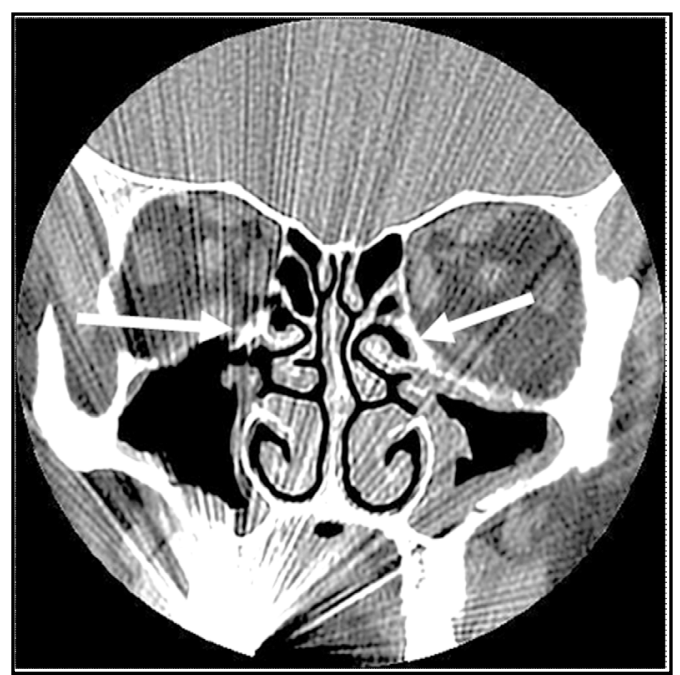

Figura 3. Cornetes medios supernumerarios. altamente prevalente. Para esta parte del estudio, se decidió excluir todas las TC de CPN de población pediátrica, ya que la neumatización de las cavidades paranasales en estos casos aún es incompleta $^{20}$. De esta forma, obtuvimos casi $4 \%$ de hipoplasia 0 aplasia de senos maxilares, y más de $14 \%$ de hipoplasia 0 aplasia de senos frontales, datos muy concordantes con la literatura ${ }^{2,6}$. La hipoplasia de senos maxilares, además de producir error diagnóstico con cuadros infecciosos al ser evaluados con radiografías simples, produce una relación anatómica aberrante con el proceso uncinado ipsilateral, dado que éste se inserta en una posición más baja de la normal. El proceso uncinado es un punto de referencia para la antrostomía maxilar, el desconocimiento de su ubicación anormal puede inducir un daño intraoperatorio inadvertido de la pared orbitaria medial durante la CEN².

Observamos $13,4 \%$ de celdillas de Onodi, la cual definimos para nuestro estudio como la proIongación hacia posterior de una celdilla etmoidal posterosuperior, de tal manera que su pared posterior está más posterior que la pared anterior de la celdilla esfenoidal vecina (Figura 4). Esta variación fue descrita por primera vez por Adolf Onodi en 1903, y su importancia radica en su estrecha relación con el nervio óptico. Por esta razón, siempre debe identificarse antes de realizar una CEN, para así evitar lesiones de nervio óptico, y eventualmente de la arteria carótida interna ${ }^{21}$. La frecuencia de esta variante varía en torno al $8 \%$ y

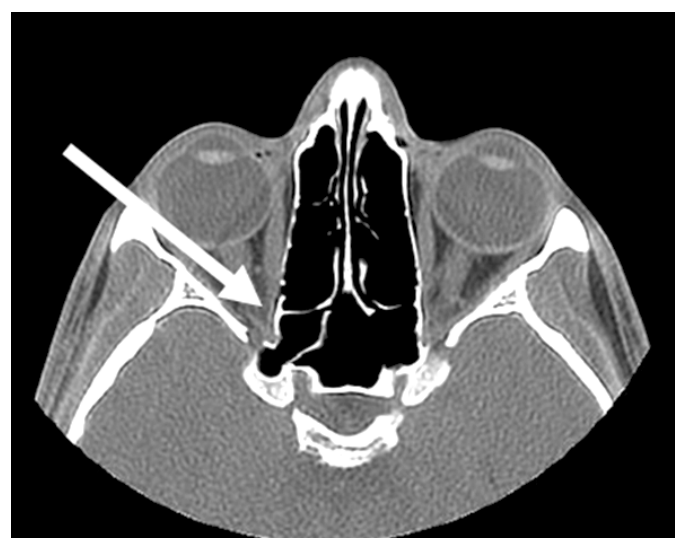

Figura 4. Celdilla esfenoetmoidal (de Onodi) derecha. Se puede apreciar íntima relación con el nervio óptico ipsilateral. 
$14 \%$, según distintas series. Pese a su relativa alta frecuencia, la compresión del nervio óptico como una complicación infecciosa de una sinusitis etmoidal posterior es algo extremadamente infrecuente ${ }^{22}$. Estudios con cadáveres han demostrado que la prevalencia de celdillas de Onodi está subestimada con el uso de TC, ya que en cadáveres se llegaría a prevalencias de hasta $60 \%{ }^{23}$.

Encontramos $10,6 \%$ de celdillas de Haller (0 infraorbitarias), cifra coincidente con lo reportado por Kennedy y Zinreich ${ }^{7}$ (Figura 5). Este es un reparo de importancia no sólo por estar implicado entre las posibles etiologías de la sinusitis maxilar unilateral recurrente, sino porque su persistencia en el posquirúrgico es una de las causas de fracaso de la antrostomía maxilar².

Si bien la emergencia de la AEA es visible en la mayoría de las TC, la trayectoria completa en la cavidad perinasal es más difícil de observar. En nuestro estudio pudimos observar la trayectoria completa en el $41,5 \%$ de los casos, cifra concordante con otros trabajos ${ }^{24,25}$. La arteria etmoidal anterior puede ser un punto de reparo importante para la CEN frontoetmoidal, dado a que las distintas trayectorias otorgarían riesgos variables a la cirugía. De los casos evaluables, se observa un predominio de lo que definimos como «trayectorias altas» (por techo etmoidal) (62\%), teóricamente más seguras durante la CEN. Sin embargo, encontramos $38 \%$ de trayectorias más bajas, las cuales podrían poseer un mayor riesgo de complicaciones $^{26}$ (Figura 6 ).

Para poder aumentar la identificación de la trayectoria completa de la AEA se requieren cortes coronales más finos que los que tuvimos disponibles (2-3 mm), lo que corresponde a una limitante en nuestro estudio.

Finalmente, decidimos estudiar la configuración del techo etmoidal. Para ello, se utilizó la clasificación de Keros, descrita por este autor en $1962^{27}$ (Figura 7), donde se mide la diferencia de altura entre la lámina cribiforme y el techo etmoidal anterior. Los techos etmoidales bajos serían los más seguros en la CEN, y existiría menor riesgo de provocar fístulas de líquido cefalorraquídeo (LCR) durante la cirugía ${ }^{11}$.

Para realizar la medición en cada caso, se buscó el corte coronal donde se puede observar la crista galli de mayor tamaño ${ }^{28}$. En nuestra revisión,

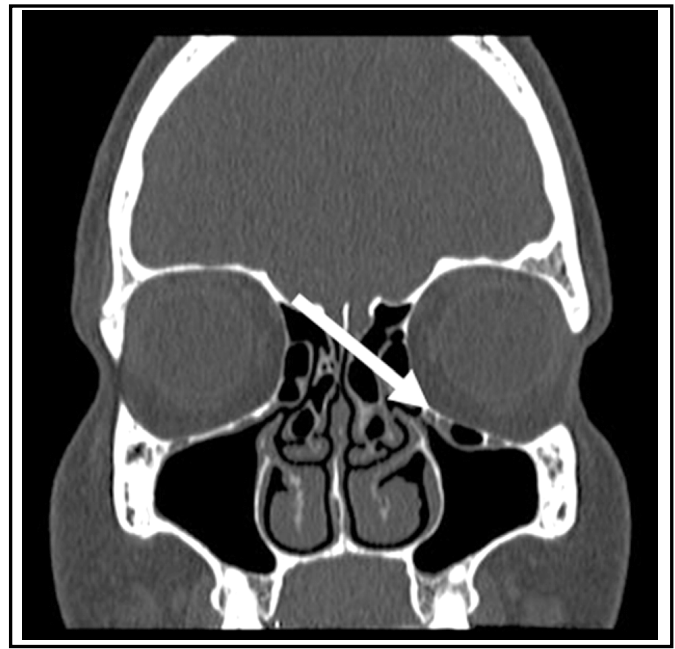

Figura 5. Corte coronal que muestra una celdilla infraorbitaria (Haller) izquierda.

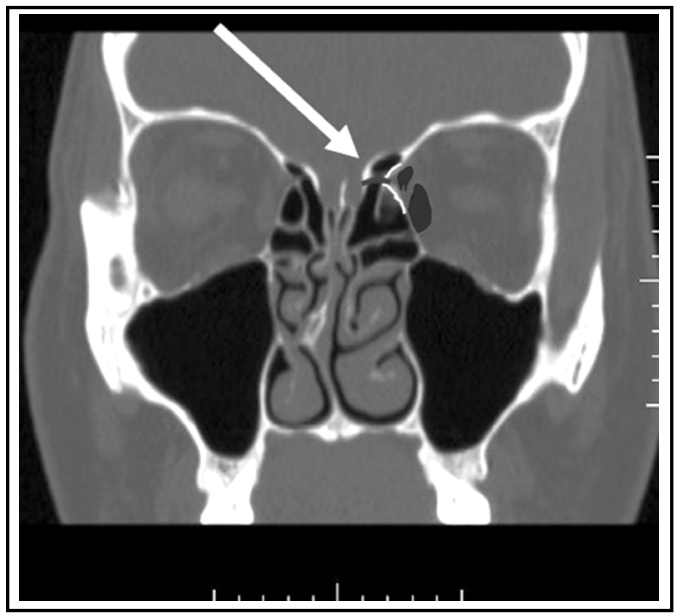

Figura 6. Corte coronal donde se esquematiza trayectoria de AEA izquierda. Se observa su salida desde la pared orbitaria medial, en relación al músculo recto medio y oblicuo superior, y su trayectoria hacia la base de cráneo anterior. En este caso, correspondería a una trayectoria baja.

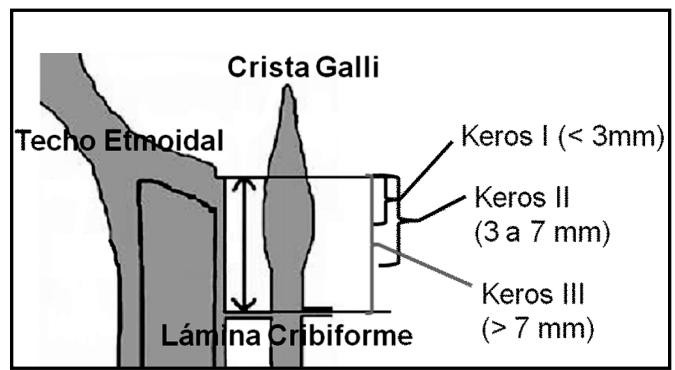

Figura 7. Clasificación de Keros. 
es llamativa la mayor frecuencia de configuración de techo etmoidal tipo Keros I (51,4\%), en comparación al predominio de techos etmoidales tipo Keros II en la mayoría de las series $(70 \%$ en el estudio de Keros) (Tabla 1). Sin embargo, existen resultados concordantes con los nuestros. En 2008 se publicó un estudio con 109 pacientes filipinos, en donde se encontró $80 \%$ de techos etmoidales Keros ${ }^{29}$.

No tenemos ninguna explicación que justifique estos hallazgos, sólo nos queda plantear como posible hipótesis, que podrían existir diferencias cefalométricas en las distintas poblaciones, y que nuestra población predominantemente hispanoamericana presentaría una configuración más bien braquicefálica, similar a poblaciones orientales ${ }^{30}$, determinando techos etmoidales más bajos, a diferencia de la mayoría de los estudios hechos con población de origen caucásico-europeo.

\section{CONCLUSIONES}

Hemos podido identificar múltiples variantes anatómicas existentes en las TC de CPN que fueron solicitados en nuestra Red de Salud, que generalmente no son informadas de rutina por los radiólogos. Por tanto, todo ORL debería entrenarse en su búsqueda para facilitar su cirugía y prever posibles complicaciones, así como solicitar al radiólogo el correcto informe de éstas según sea necesario.

Nuestros resultados son en general comparables con los publicados en la literatura extranjera.

El Agger Nassi sería en la práctica la «variante» anatómica más frecuente.

Existe un predominio de techos etmoidales tipo Keros I en los casos analizados, a diferencia de la mayoría de las publicaciones internacionales.

La determinación de la trayectoria completa de la AEA requiere del análisis de cortes radiológicos más finos que los que tuvimos disponibles en nuestro estudio, lo que explica que el número de casos analizables en nuestra revisión fuera escaso. Este podría ser un argumento de peso para exigir estudios radiológicos con cortes más finos que los habitualmente realizados.

La escasa cantidad de TC de CPN con cortes sagitales disponibles no nos permitió realizar el análisis de otras variaciones, tales como las del complejo nasofrontal. Esto recalca la necesidad de solicitar los cortes sagitales en forma rutinaria, sobre todo si se sospecha patología del seno frontal.

\section{BIBLIOGRAFÍA}

1. MesserkLIng $W$. On the drainage of the normal frontal sinus of man. Acta Otolaryngol (Stockh) 1967; 63: 176-81.

2. KantaRCI M. Remarkable anatomic variations in paranasal sinus region and their clinical importance. European Journal of Radiology 2004; 50: 296-302.

3. Calmoun KH, WagGenspack GA. CT evaluation of the paranasal sinuses in symptomatic and asymptomatic populations. Otolaryngol Head Neck Surg 1991; 104: 480-3.

4. Allan Keast, BSc, MB, ChB, Sofie Yelavich, MB, ChS, Patrick Dawes, MB, BS, FRCS, and Brett Lyons, MB, $\mathrm{CHB}_{\mathrm{H}}$. Anatomical variations of the paranasal sinuses in Polynesian and New Zealand European computerized tomography scans. OtolaryngologyHead and Neck Surgery 2008; 139: 216-21.

5. ARSLAN H. Anatomic variations of the paranasal sinuses: CT examination for endoscopic sinus surgery. Auris Nasus Larynx 1999; 26(1): 39-48.

6. J. Jiménez-Castellanos. Anatomical variations in the human paranasal sinus region studied by $C T$. $J$ Anat 2000; 197: 221-7.

7. KenNedY DV, ZINREICH SJ. Functional endoscopic approach to inflammatory sinus disease: current prospectives and technique modifications. Am J Rhinol 1988; 2: 89-96.

8. Harar RP, Chadha NK, Rogers $G$. The role of septal desviation in adult chronic rhinosinusitis: a study of 500 patients. Rhinology 2004; 42 (3): 126-30.

9. Collet S, Bertrand B, Cornu S, Eloy P, Rombaux P. Is septal deviation a risk factor for chronic sinusitis? Review of literature. Acta Otorhinolaryngol Belg 2001; 55(4): 299-304.

10. Hasan Yasan, MD, Harun Dogru, MD, Bahattin Baykal, MD, FehmI Doner, MD, and Mustafa Tuz. What is the relationship between chronic sinus disease and isolated nasal septal deviation? Oto-Head and Neck Surgery 2005; 133: 190-3.

11. NouRael SA. Variations in paranasal sinus anatomy: implications for the pathophysiology of chronic rhinosinusitis and safety of endoscopic sinus 
surgery. J Otolaryngol Head Neck Surg 2009; 38(1): 32-7.

12. AgulleRA M. Variaciones Anatómicas Rinosinusales Encontradas por Tomografía Computarizada. Sociedad Boliviana de Radiología.(www.sbrbolivia.com/ revista) Vol 3 N01, 17-21.

13. Khanobthamchal K, Shankar L, Hawke M, Bingham B. The secondary middle turbinate. J Otolaryngol 1991; 20(6): 412-3.

14. Homsioglou E, Balatsouras DG, Alexopoulos G, Kaberos A, Katotomichelakis $M$, Danielides $V$. Pneumatized superior turbinate as a cause of headache. Head Face Med 2007; 3: 3.

15. Clerico, Dean M. MD. Pneumatized Superior Turbinate as a Cause of Referred Migraine Headache. Laryngoscope 1996; 106(7): 874-9.

16. Mehle M MD FACS, Kremer P RN. Sinus CT Scan Findings in «Sinus Headache» Migraineurs. Headache 2008; 48: 67-71.

17. Gordon Shields, Hadi Seikaly, Matthew LeBoeuf, Faustino Guinto, Herve LeBoeuf, Thomas Pincus, Karen Calnoun. Correlation Between Facial Pain or Headache and Computed Tomography in Rhinosinusitis in Canadian and U.S. Subjects. Laryngoscope 2003; 113: 943-5.

18. M ABu-BAKRA, NS Jones. Prevalence of nasal mucosal contact points in patients with facial pain compared with patients without facial pain. The Journal of Laryngology \& Otology 2001; 115: 629-32.

19. AK Bieger-Farahn, J Nichani and DJ Willatt. Nasal septal mucosal contact points: associated symptoms and sinus CT scan scoring. Clin Otolaryng 2004; 29: 165-8.

20. Barghouth G, Prior JO, Lepori D, Duvoisin B, SCHnyder P, Gudinchet F. Paranasal sinuses in children: size evaluation of maxillary, sphenoid, and frontal sinuses by magnetic resonance imaging and proposal of volume index percentile curves. Eur Radiol 2002; 12 (6): 1451-8.

21. Yanagisawa E, Weaver EM, Ashikawa R. The Onodi (sphenoethmoid) cell. Ear Nose Throat J 1998; 77 : 578-80.

22. Driben JS, Bolger We, Robles HA, Cable B, Zinreich SJ. The reliability of computerized tomographic detection of the Onodi (Sphenoethmoid) cell. Am J Rhinol 1998; 12(2): 105-11.
23. Thanaviratananich S, Chaisimamongkol K, Kraitrakul S, ET AL. The prevalence of an Onodi cell in adult Thai cadavers. Ear Nose Throat J 2003; 82: 200-4.

24. Souza SA, Souza MM, Gregório LC, Auzen S. Anterior ethmoidal artery evaluation coronal CT scans. Braz J Otorhinolaryngol 2009; 75(1): 101-6.

25. McDonald SE, Robinson PJ, NuñEz DA. Radiological anatomy of the anterior ethmoidal artery for functional endoscopic sinus surgery. J Laryngol Otol 2008; 122 (3): 264-7.

26. Yang YX, Lu QK, LiaO JC, Dang RS. Morphological characteristics of the anterior ethmoidal artery in ethmoid roof and endoscopic localization. Skull Base 2009; 19 (5): 311-7.

27. KEROS P. On the practical value of differences in the level of the lamina cribrosa of the ethmoid. $Z$ Laryngol Rhinol Otol 1962; 41: 809-13.

28. MeYers, Robert M. Interpretation of Anatomic Variations of Computed Tomography Scans of the Sinuses: A Surgeon's Perspective. Laryngoscope 1998; 108: $422-5$.

29. Josefino G. HeRnÁndez. Radiographic Analysis of the Ethmoid Roof based on Keros Classification among Filipinos. Philipp. J Otolaryngol Head Neck Surg 2008; 23 (1): 15-9.

30. Iol H, Nakata S, Nakasima A, Counts AL. Comparison of cephalometric norms between Japanese and Caucasian adults in antero-posterior and vertical dimension. Eur J Orthod 2007; 29 (5): 493-9.

31. Bolger W. Paranasal Sinus Bony Anatomic Variations and Mucosal Abnormalities: CT Analysis for Endoscopic Sinus Surgery. Laryngoscope 1991; 101(1): 56-64.

32. BASIC N. Computed tomographic imaging to determine the frequency of anatomical variations in pneumatization of the ethmoid bone. Eur Arch Otorhinolaryngol 1999; 256(2): 69-71.

33. Gonzálzz-Rosado G. Relación de la profundidad y morfología del techo etmoidal por TC con patología de seno etmoidal y/o frontal. Anales de Radiología México 2006; 2: 147-52.

34. Aksungur EH, Bicakcl K, Inal M, AкGül E, Binokay F, Aydogan B, OGuz M. CT demonstration of accessory nasal turbinates: secondary middle turbinate and bifid inferior turbinate. Eur J Radiol 1999; 31 (3): 174-6. 